\title{
Accidental Degeneracy of Hydrogen Atom Revisited
}

\section{Su WC*}

Department of Physics, National Chung-Cheng University, Taiwan

\begin{abstract}
We examine accidental symmetry of hydrogen-like atoms analytically in spherical coordinates. Some unfamiliar but notable functional relations of the radial eigenfunctions of system's Hamiltonian are constructed, for both discrete and continuous energy eigenvalues. Analytical similarity of the symmetry between two-dimensional and threedimensional hydrogen atoms are therefore demonstrated.
\end{abstract}

Keywords: Quantum mechanics; Accidental symmetry; Hydrogen atom

\section{Introduction}

In quantum mechanics, it is generally believed that in static sense the geometric symmetry of a Hamiltonian is responsible for the degeneracy of energy levels. If this situation does not occur, we customarily claim that there is an accidental symmetry that causes the accidental degeneracy of energy levels. Sometimes, the term "hidden symmetry" is also used as the synonym of accidental symmetry in the literature. In non-relativistic quantum mechanics, the well known example of accidental degeneracy is the hydrogen atom with Coulomb $1 / r$ potential, in which the degree of degeneracy for a bound-state of principal quantum number $n$ is equal to $n^{2}$ but not the expected $2 l+1$ value as anticipated from a generic central potential. Here, we ignore the spin degree of freedom.

Pauli was the pioneer who introduced the Runge-Lenz operator of the hydrogen atom to explain the accidental degeneracy in the energy levels [1]. The mystery of that accidental symmetry was then disclosed by Fock, who found that all the bound-state eigenfunctions in ordinary three-dimensional space can be translated into so(4) hyperspherical harmonic functions constructed in a fictitious fourEuclidean space [2]. As a result, eigenfunctions with the same value of $n$, but different orbital angular momentum quantum number $l$, can be transformed into one another by rotations in this four-dimensional mathematical space. Later, Bargmann gave a Lie algebraic approach of the so(4) hyperspherical symmetry to demonstrate that the accidental degeneracy of the hydrogen atom is indeed due to a hidden symmetry, which is not completely realized in the original three-Euclidean space [3].

To be more specific, the Hamiltonian of hydrogen atom in ordinary three-dimensional space is of the form $H=\frac{\mathbf{p}^{2}}{2 \mu}-\frac{\kappa}{r}$, yielding the time-independent Schrödinger equation $H \phi_{n}=E_{n} \phi_{n}$. Obviously, the potential energy and kinetic energy separately obey the $s o(3)$ rotational symmetry. Therefore, the so-called so(4) hyperspherical symmetry of the bound-state eigenfunctions is definitely not a symmetry of the potential energy, nor is it a symmetry of the kinetic energy. It is a symmetry of the entire Hamiltonian itself! In such away, rotations of so(4) hyperspherical symmetry will leave the Hamiltonian $H$ invariant, hence the energy eigenvalues $E_{n}$, too.

We remark that in reality the so(4) hyperspherical symmetry is not the maximal symmetry the Hamiltonian $H$ can possess when it is in motion. It was discovered in the 60's that the time-dependent Schrodinger equation $i \hbar \frac{\partial}{\partial t} \psi=H \psi$ admits an even larger "dynamical symmetry" than the accidental symmetry realized by the corresponding time-independent one [4-11]. The reason is that in time-dependent Schrödinger equation the energy eigenvalues $E_{n}$ can be treated as a variable. So, there exists an additional spectrum-generating so $(2,1)$ symmetry that can be used to vary among different energy levels $[12,13]$. In effect, this $s o(2,1)$ symmetry is combined with the originally so(4) hyperspherical symmetry to form an irreducible representation of an extended $s o(4,2)$ dynamical symmetry, normally known as the conformal symmetry. For complete tabulations of $s o(4,2)$ dynamical symmetry of the hydrogen atom $[14,15]$.

In this paper, we revisit accidental symmetry of hydrogen-like atoms in the framework of time-independent Schrödinger equation. Some analytical properties associated with that symmetry are explored. Rescaled Runge-Lenz vectorial operator is introduced, to simplify the presentations. The algebra of the symmetry is briefly reviewed. Differential equations of the eigenstates, when acted on by ladder-type generators of the algebra, are inspected. Analytical properties of the ladder generators constructed in dimensionless spherical coordinates are examined, particularly for both bound states and continuous states. This results in the discovery of some unfamiliar nevertheless notable functional relations for the radial eigenfunctions of the Hamiltonian. Moreover, similar analytical properties of the radial eigenfunctions in two-dimensional hydrogen atoms are subsequently demonstrated.

The paper is organized as follows. In section 2, we briefly review the main algebraic structures of the accidental symmetry. In section 3 , we analyze analytical properties of accidental symmetry and deduce several functional relations for the radial eigenfunctions. We show analytical similarity between two-dimensional and three-dimensional hydrogen atoms in section 4 . Finally, section 5 contains the conclusion.

\section{Symmetry of Coulomb Potential}

In this section, we briefly review for the purpose of completeness the main algebraic structures of a charged particle in the presence of Coulomb $1 / r$ potential. The Hamiltonian that we will consider is the hydrogen-like atom of atomic number $Z$, which takes the same form

*Corresponding author: Su WC, Professor, Department of Physics, Nationa Chung-Cheng University, Chia-Yi 621, Taiwan, Tel: +866-5-2720411; E-mail: physuw@ccu.edu.tw

Received September 21, 2017; Accepted October 31, 2017; Published November 03, 2017

Citation: Su WC (2017) Accidental Degeneracy of Hydrogen Atom Revisited. J Phys Math 8: 253. doi: 10.4172/2090-0902.1000253

Copyright: (c) 2017 Su WC. This is an open-access article distributed under the terms of the Creative Commons Attribution License, which permits unrestricted use, distribution, and reproduction in any medium, provided the original author and source are credited. 
as: $H=\frac{\mathbf{p}^{2}}{2 \mu}-\frac{\kappa}{r}$, in which $p$ is the momentum operator, $\mu$ the reduced mass and $\kappa=\frac{Z e^{2}}{4 \pi \varepsilon_{0}}=Z \alpha \hbar c$, with $\alpha=\frac{e^{2}}{4 \pi \varepsilon_{0} \hbar c}$ the fine structure
constant.

It is known from textbooks of quantum mechanics that the algebra of accidental symmetry of the Hamiltonian $H$ is generated by the angular momentum operator $\mathbf{L}=\left(L_{x}, L_{y}, L_{z}\right)$ and the so-called RungeLenz vectorial operator $\mathbf{R}=\left(R_{x}, R_{y}, R_{z}\right)$ given by [16-18]:

$$
\mathbf{R}=\frac{1}{2 \mu \kappa}(p \times L-L \times p)-\frac{r}{r}
$$

The commutation relations among the two sets of $\mathbf{L}$ and $\mathbf{R}$ operators become simplified, if we are tempted to work on the representation of eigenstates of the Hamiltonian. In this way, $H$ can be directly replaced by the energy eigenvalue $E$ of the corresponding eigenstate considered. Owing to this fact, let us define the rescaled Runge-Lenz operator that has the dimensions of $\hbar$, similar to the angular momentum $\mathbf{L}$ does, as:

$$
\mathbf{A}=\sqrt{( \pm) \frac{\mu \kappa^{2}}{2 E}} \mathbf{R} .
$$

In eqn. (2), because the eigenvalues $E$ of $H$ contains a discrete set and a continuous energy variable, we are led to distinguish these two different spectra by introducing the $( \pm)$ symbol. It means that the boldfaced plus sign in the parenthesis stands for the case of continuous states $(E>0)$. whereas the boldfaced minus sign for that of bound states $(E<0)$. Thereinafter, we shall adopt this neat convention of the symbol $( \pm)$ throughout the paper, unless it is otherwise emphasized. That is to say, if an equation appears to not have any $( \pm)$ symbol, it simply means that this equation holds true for both $E>0$ and $E<0$ cases.

In terms of $\mathbf{L}$ and $\mathbf{A}=\left(A_{x}, A_{y}, A_{z}\right)$, the algebra of Coulomb symmetry can be derived in a straightforward manner as (for $i, j, k=x, y, z=1,2,3)$

$$
\begin{aligned}
& {\left[L_{i}, L_{j}\right]=\mathrm{i} \hbar \varepsilon_{i j k} L_{k},} \\
& {\left[L_{i}, A_{j}\right]=\mathrm{i} \hbar \varepsilon_{i j k} A_{k},} \\
& {\left[A_{i}, A_{j}\right]=-( \pm) \mathrm{i} \hbar \varepsilon_{i j k} L_{k} .}
\end{aligned}
$$

The convention of bold-plus for $E<0$ and bold-minus for $E<0$ in eqn. (5) is understood. Needless to say, the commutation relations in above equations describe an $s o(3,1)$ symmetry for $E>0$ and an $s o(4)$ symmetry for $E<0$. In is noted that for $E<0$ the $s o(4)$ algebra may be translated to $s o(3) \times s o(3)$ by transforming $\mathbf{L}$ and $\mathbf{A}$ into $\mathbf{J}_{ \pm}=\frac{1}{2}(\mathbf{L} \pm \mathbf{A})$ which furthermore admits another realization in terms of two sets of bosonic spin operators [19-21]. As a byproduct, eqn. (4) implies that the Runge-Lenz operator A transforms in the spin-one (vector) representation under three-dimensional spatial rotations.

In quantum mechanics, the symmetry of a system is encoded in the maximal set of mutually commuting operators that includes the Hamiltonian inside of it. The set is responsible for the degeneracy of energy spectrum and results in various constants of motion in dynamics. For the accidental symmetry of hydrogen atoms we considered, the commuting set can take, for example, to be $\left\{H, \mathbf{L}^{2}, L_{z}, \mathbf{A}^{2}\right\}$. Hence, let us designate the simultaneous normalized eigenstates of these commuting operators by $\mid l, m>_{n}$ with the corresponding energy eigenvalues

$$
E=( \pm) \frac{1}{2} \mu c^{2} \frac{(Z \alpha)^{2}}{n^{2}}
$$

Depending on the sign of $E$, we demand that $n \in R^{+}$be a real positive variable for the bold-plus $(E>0)$ ase and $n \in Z^{+}$a positive integer for the bold-minus $(E<0)$ case. Note that, for the $E>0$ case, the continuous variable $n$ is related to the wave number $k$ of positive energy eigenvalue $E=\frac{\hbar^{2} k^{2}}{2 \mu}$ at infinity by $n=\frac{Z \alpha \mu c}{\hbar k}$.

With such a choice, the eigenvalue equations for $\mathbf{L}^{2}$ and $L_{z}$ are the standard ones found in quantum mechanical textbooks, while that for operator $\mathbf{A}^{2}$ can be established from the operator identity: $\mathbf{A}^{2}=( \pm)\left(\mathbf{L}^{2}+\hbar^{2}\right)+\hbar^{2} n^{2}$. It is

$$
\mathbf{A}^{2}\left|l, m>_{n}=\hbar^{2}\left[( \pm)\left(l^{2}+l+1\right)+n^{2}\right]\right| l, m>_{n} .
$$

To further analyze algebraic properties of the symmetry, it proves convenient to introduce ladder-type operators for both $\mathbf{L}$ and $\mathbf{A}$ by $L_{ \pm} \equiv L_{x} \pm \mathrm{i} L_{y}$ and $A_{ \pm} \equiv A_{x} \pm \mathrm{i} A_{y}$, from which we immediately arrive at the operator identities: $\mathbf{L}^{2}=L_{ \pm} L_{\mp}+L_{z}^{2} \mp \hbar L_{z}$ and $\mathbf{A}^{2}=A_{ \pm} A_{+}+A_{z}^{2} \pm( \pm) \hbar L_{z}$. As a result, the commutation relations in eqns. (3)-(5) can be reformulated in terms of these ladder-type operators into:

$$
\begin{aligned}
& {\left[L_{+}, L_{-}\right]=2 \hbar L_{z}, \quad\left[L_{z}, L_{ \pm}\right]= \pm \hbar L_{ \pm},} \\
& {\left[L_{ \pm}, A_{+}\right]= \pm 2 \hbar A_{z}, \quad\left[L_{z}, A_{ \pm}\right]= \pm \hbar A_{ \pm}, \quad\left[A_{z}, L_{ \pm}\right]= \pm \hbar A_{ \pm},} \\
& {\left[A_{+}, A_{-}\right]=-( \pm) 2 \hbar L_{z}, \quad\left[A_{z}, A_{ \pm}\right]=\mp( \pm) \hbar L_{ \pm} .}
\end{aligned}
$$

some other commutators not shown above are trivially zero.

The action of ladder-type operators on the designated eigenstates is again standard for $\mathbf{L}$, that is, $L_{z}\left|l, m>_{n}=\hbar m\right| l, m>_{n}$ and $L_{ \pm}\left|l, m>_{n}=\hbar \mathrm{C}^{ \pm}(l, m)\right| l, m>_{n}$, with $\quad \mathrm{C}^{ \pm}(l, m)=\sqrt{(l \mp m)(l \pm m+1)}$. As regards to those for $\mathbf{A}$, we have already in mind that it is a spin-one tensor operator. Therefore, according to general rules for addition of angular momenta, we may formally write the corresponding equations by

$$
\begin{aligned}
& \frac{1}{\hbar} A_{+}\left|l, m>_{n}=\alpha_{+}(l, m)\right| l+1, m+1>_{n}+\beta_{+}(l, m) \mid l-1, m+1>_{n}, \\
& \frac{1}{\hbar} A_{-}\left|l, m>_{n}=\alpha_{-}(l, m)\right| l+1, m-1>_{n}+\beta_{-}(l, m) \mid l-1, m-1>_{n}, \\
& \frac{1}{\hbar} A_{z}\left|l, m>_{n}=\alpha_{0}(l, m)\right| l+1, m>_{n}+\beta_{0}(l, m) \mid l-1, m>_{n} .
\end{aligned}
$$

here, the coefficients $\alpha_{i}(l . m)$ and $\beta_{i}(l . m)$ (for $i=+,-, 0$ ) also depend on the parameter $n$, though it is suppressed in the expressions.

The coefficients $\alpha_{i}(l . m)$ and $\beta_{i}(l . m)$ can be directly determined from the commutation relations listed in eqns. (9) and (10). For instance, applications of the first commutator of eqn. (9) give rise to the following coefficients relations

$$
\alpha_{ \pm}(l, m)=\mp \sqrt{\frac{l \pm m+2}{l \mp m+1}} \alpha_{0}(l, m), \quad \beta_{ \pm}(l, m)= \pm \sqrt{\frac{l \mp m-1}{l \pm m}} \beta_{0}(l, m) .
$$

Furthermore, other commutators or identities will give the explicit forms for both $\alpha_{0}(l . m)$ and $\beta_{0}(l . m)$. After some manipulations, we get

$$
\alpha_{0}(l, m)=\left[\frac{\left(n^{2}+( \pm)(l+1)^{2}\right)\left((l+1)^{2}-m^{2}\right)}{(2 l+1)(2 l+3)}\right]^{1 / 2}, \quad \beta_{0}(l, m)=\alpha_{0}(l-1, m) .
$$

The relations below reflecting the symmetry of Coulomb $1 / r$ potential can be deduced easily

$$
\alpha_{0}(l,-m)=\alpha_{0}(l, m), \quad \alpha_{-}(l, m)=-\alpha_{+}(l,-m) .
$$

Likewise, we have $\beta_{-}(l, m)=-\beta_{+}(l,-m)$. Let us remind once more that the boldfaced $( \pm$ ) in eqn. (15) is for $E>0$ and $E<0$ cases, respectively. 


\section{Properties of the Symmetry}

The algebraic structures of Coulomb potential presented in section 2 enable us to investigate some analytical properties of the eigenstates $\mid l, m>_{n}$ in the spherical coordinates, $\mathbf{r}=(r, \theta, \varphi)$. Before going into the details, let us make one more variable change to simplify our presentations. Instead of $\mathbf{r}$, we define the dimensionless position vector by $\boldsymbol{\rho} \equiv \tilde{\alpha} \mathbf{r}=(\rho, \theta, \phi)$ for $\tilde{\alpha}=\frac{2 \mu \kappa}{n \hbar^{2}}$, so that the eigenfunctions we are interested will be denoted by $\psi_{n l m}(\boldsymbol{\rho})=<\boldsymbol{\rho}|l, m\rangle_{n}$. Since $\mathbf{L}^{2}$ is still a good observable, it is again legal to separate $\psi_{n l m}(\rho)$ into the radial $R_{n l}(\rho)$ and angular $Y_{l}^{m}(\theta, \phi)$ parts as

$$
\psi_{n l m}(\rho)=A_{n l} R_{n l}(\rho) Y_{l}^{m}(\theta, \phi),
$$

where $A_{l n}$ is the normalization factor that depends on both $l$ and $n$.

In the dimensionless spherical coordinates $\boldsymbol{\rho}=(\rho, \theta, \varphi)$, the RungeLenz operators $\left(A_{z}, A+, A-\right)$ are expressed as follows

$$
\begin{aligned}
& \frac{1}{\hbar} A_{z}=2 \cos \theta\left[\partial_{\rho}+\frac{n}{2}-\frac{1}{\rho} \frac{\mathbf{L}^{2}-L_{z}^{2}}{\hbar^{2}}\right]+e^{i \phi} \sin \theta\left[\partial_{\rho}-\frac{1}{\rho}\left(1+\frac{L_{z}}{\hbar}\right)\right] L_{-}-e^{-i \phi} \sin \theta\left[\partial_{\rho}-\frac{1}{\rho}\left(1-\frac{L_{z}}{\hbar}\right) L_{+},\right. \\
& \frac{1}{\hbar} A_{ \pm}= \pm 2 \cos \theta \partial_{\rho} \frac{L_{ \pm}}{\hbar}-2 e^{ \pm i \phi} \sin \theta\left[\partial_{\rho}\left(1 \pm \frac{L_{z}}{\hbar}\right)+\frac{n}{2}-\frac{1}{\rho} \frac{\mathbf{L}^{2}}{\hbar^{2}}\right],
\end{aligned}
$$

where $\partial_{\rho}=\frac{\partial}{\partial \rho}$ is used.

Having found eqns. (18) and (19), we now analyze on what analytical constraints that are set by algebraic eqs. (11) to (13) are imposed on the eigenfunctions $\psi_{n l m}(\boldsymbol{\rho})$. Let us answer this question by first taking the differential operator $\frac{1}{\hbar} A_{z}$ and acting it on the state $\psi_{n l m}(\boldsymbol{\rho})$. Bearing in mind in advance with the algebraic eqn. (13), we would expect the neat result below

$$
\frac{1}{\hbar} A_{z} \psi_{n l m}=\alpha_{0}(l, m) A_{n, l+1} R_{n, l+1} Y_{l+1}^{m}+\beta_{0}(l, m) A_{n, l-1} R_{n, l-1} Y_{l-1}^{m} .
$$

Now, back to the corresponding differential operation the computation is straightforward. We obtain

$$
\begin{aligned}
& \frac{1}{\hbar} A_{z} \psi_{n l m}=2 A_{n l}\left\{\left[\partial_{\rho}+\frac{n}{2}-\frac{l(l+1)-m^{2}}{\rho}\right]\left(\mathrm{D}_{+}^{0}(l, m) Y_{l+1}^{m}+\mathrm{D}_{-}^{0}(l, m) Y_{l-1}^{m}\right)-\frac{1}{2}\left[\partial_{\rho}-\frac{m}{\rho}\right] \mathrm{C}^{-}(l, m)\right. \\
& \left(\mathrm{D}_{+}^{+}(l, m-1) Y_{l+1}^{m}+\mathrm{D}_{-}^{+}(l, m-1) Y_{l-1}^{m}\right)+\frac{1}{2}\left[\partial_{\rho}+\frac{m}{\rho}\right] \mathrm{C}^{+}(l, m)\left(\mathrm{D}_{+}^{-}(l, m+1) Y_{l+1}^{m}+\mathrm{D}_{-}^{-}(l, m+1) Y_{l-1}^{m}\right) ; R_{n l},
\end{aligned}
$$

where the coefficients $\mathrm{D}_{i}^{j}$ (for $i, j=+,-, 0$ ) come from the combination of a factor of $\cos \theta$ or $e^{ \pm i \phi} \sin \theta$ with the $Y_{l}^{m}$ factor. Eqn. (21) is not very nice-looking, however it can be simplified. For instance, in the second and third lines of that equation the product of $\mathrm{C}^{ \pm}$and $\mathrm{D}_{i}^{j}$ can be arranged into either $\mathrm{D}_{+}^{0}(l, m)$ or $\mathrm{D}_{-}^{0}(l, m)$, that are subsequently related to the coefficients $\alpha_{0}(l . m)$ and $\beta_{0}(l . m)$ given in eqn. (15). See Appendix A for the detailed relations and computations of these coefficients.

$$
\begin{aligned}
& \text { After the arrangement, we are left with } \\
& \frac{1}{\hbar} A_{z} \psi_{n l m}=\alpha_{0}(l, m) \frac{-2 A_{n l}}{\sqrt{n^{2}+( \pm)(l+1)^{2}}}\left[(l+1) \partial_{\rho}+\frac{n}{2}-\frac{l(l+1)}{\rho}\right] R_{n l} Y_{l+1}^{m}+ \\
& \beta_{0}(l, m) \frac{-2 A_{n l}}{\sqrt{n^{2}+( \pm) l^{2}}}\left[-l \partial_{\rho}+\frac{n}{2}-\frac{l(l+1)}{\rho}\right] R_{n l} Y_{l-1}^{m} .
\end{aligned}
$$

remember that eqn. (22) must be identical to eqn. (20). For such assertion to hold, there are in general two alternative options in the discussion of the Coulomb $1 / r$ potential. It depends on whether $E<0$ or $E>0$, Let us discuss them separately.

1. The case of $E<0$. The spectrum of negative energy values is discrete and depends on the principal quantum number $n$. The degree of degeneracy of the $n$-th energy level is $n^{2}$, for $l=0$ to $n-1$ and $m=-l$ to $l$. This case corresponds to take the boldfaced minus in the symbol $( \pm)$ in eqn. (22), in which the normalization factor $A_{n l}$ is normally chosen to satisfy the recurrence relation

$$
A_{n l}=\sqrt{n^{2}-(l+1)^{2}} A_{n, l+1}=\frac{1}{\sqrt{n^{2}-l^{2}}} A_{n, l-1} .
$$

It hence implies the general expression for $A_{n l}$ as

$$
\left[(l+1) \partial_{\rho}+\frac{n}{2}-\frac{l(l+1)}{\rho}\right] R_{n l}(\rho)=-\frac{1}{2} R_{n, l+1}(\rho),
$$

where $A_{n 0}$ is an $n$ dependent constant, not completely fixed by the purely algebraic method. In eqn. (24), the product is replaced by unity for $l=0$.

Turn to eqn. (22), the recurrence relation (23) additionally determine two first-order linear differential equations that are satisfied by the radial functions $R_{n l}(\rho)$. They are respectively

$$
\begin{aligned}
& {\left[(l+1) \partial_{\rho}+\frac{n}{2}-\frac{l(l+1)}{\rho}\right] R_{n l}(\rho)=-\frac{1}{2} R_{n, l+1}(\rho),} \\
& {\left[-l \partial_{\rho}+\frac{n}{2}-\frac{l(l+1)}{\rho}\right] R_{n l}(\rho)=-\frac{1}{2}\left(n^{2}-l^{2}\right) R_{n, l-1}(\rho) .}
\end{aligned}
$$

Now, the radial eigenfunctions for $E<0$ is known to be

$$
R_{n l}(\rho)=\rho^{l} e^{-\rho / 2} L_{n-l-1}^{2 l+1}(\rho),
$$

where $L_{\alpha}^{\beta}(\rho)$ are the associated Laguerre polynomials. When substituting $R_{n l}(\rho)$ into both (25) and (26) and making use of the identity $\partial_{x} L_{\alpha}^{\beta}(x)=-L_{\alpha-1}^{\beta+1}(x)$, we arrive at two unfamiliar but notable functional relations of $L_{\alpha}^{\beta}(\rho)$

$$
\begin{aligned}
& -\frac{\rho}{2} L_{n-l-2}^{2 l+3}(\rho)=-(l+1) L_{n-l-2}^{2 l+2}(\rho)+\frac{1}{2}(n-l-1) L_{n-l-1}^{2 l+1}(\rho), \\
& -\frac{n^{2}-l^{2}}{2 \rho} L_{n-l}^{2 l-1}(\rho)=l L_{n-l-2}^{2 l+2}(\rho)+\left(\frac{n+l}{2}-\frac{l(2 l+1)}{\rho}\right) L_{n-l-1}^{2 l+1}(\rho) .
\end{aligned}
$$

Here, both equations can be verified by tools of computational software, such as the Mathematica. By the way, the factor $A_{n 0}=\left[\frac{\tilde{\alpha}^{3}}{2 n^{2}}\right]^{1 / 2}$ is fixed by the orthonormality condition: $\int \psi_{n^{\prime} l m}^{*}(\rho) \psi_{n l m}(\rho) d^{3} r=\delta_{n n^{\prime}}$, where $\boldsymbol{\rho}=\tilde{\alpha} \mathbf{r}$ and $\tilde{\alpha}=\frac{2 \mu \kappa}{n \hbar^{2}}$ have been defined previously.

2. The case of $E>0$. The spectrum of positive energy eigenvalues is continuous from zero to infinity. Each of these eigenvalues is of infinite degeneracy, with $l$ running from 0 to $\infty$ and with all possible values of $m$ for a given $l$. We pick the boldfaced plus in the symbol ( \pm ) in eqn. (22). To be consistent with the conventional radial eigenfunctions for continuous spectrum, we need to select this relation fulfilled by the factor $A_{n l}$

$$
A_{n l}=\frac{1}{\sqrt{n^{2}+(l+1)^{2}}} A_{n, l+1}=\sqrt{n^{2}+l^{2}} A_{n, l-1},
$$

which inferring that

$$
A_{n l}=A_{n 0} \prod_{s=1}^{l}\left(n^{2}+s^{2}\right)^{1 / 2},
$$

where $A_{n 0}$ is another $n$ dependent constant, not completely fixed by algebraic methods. Similarly, the product have to be replaced by unity for $l=0$.

In the same vein, the recurrence relation (30) additionally determine two first-order linear different equations of the radial functions $R_{n l}(\rho)$. They are: 


$$
\begin{aligned}
& {\left[(l+1) \partial_{\rho}+\frac{n}{2}-\frac{l(l+1)}{\rho}\right] R_{n l}(\rho)=-\frac{1}{2}\left(n^{2}+(l+1)^{2}\right) R_{n, l+1}(\rho),} \\
& {\left[-l \partial_{\rho}+\frac{n}{2}-\frac{l(l+1)}{\rho}\right] R_{n l}(\rho)=-\frac{1}{2} R_{n, l-1}(\rho) .}
\end{aligned}
$$
form

For the continuous spectrum, the radial eigenfunctions is of the

$$
R_{n l}(\rho)=\frac{1}{(2 l+1) !} \rho^{l} e^{\mathrm{i} \rho / 2} F(l+1-\mathrm{i} n, 2 l+2 ;-\mathrm{i} \rho),
$$

where $F(\alpha ; \beta ; z)$ for $\mathrm{z}$ a complex variable are the confluent hypergeometric functions. When substituting (34) into both (25) and (26) and making use of the identity $\partial_{z} F(\alpha, \beta ; z)=\frac{\alpha}{\beta} F(\alpha+1, \beta+1 ; z)$, we obtain two more unfamiliar functional relations

$$
\begin{aligned}
& \frac{l+1+\mathrm{i} n}{(2 l+2)(2 l+3)} z F(l+2-\mathrm{i} n, 2 l+4 ; z)= \\
& F(l+2-\mathrm{i} n, 2 l+3 ; z)-F(l+1-\mathrm{i} n, 2 l+2 ; z), \\
& F(l-\mathrm{i} n, 2 l ; z)=\left(1-\frac{l+\mathrm{i} n}{2 l(2 l+1)} z\right) F(l+1-\mathrm{i} n, 2 l+2 ; z)+ \\
& \frac{l+1-\mathrm{i} n}{(2 l+1)(2 l+2)} z F(l+2-\mathrm{i} n, 2 l+3 ; z),
\end{aligned}
$$

where $z=-i p$ Combining eqns. (35) and (36) renders another functional relation similar to that of eqn. (35). It is

$$
\begin{aligned}
& \frac{l+1-\mathrm{i} n}{(2 l+1)(2 l+2)} z F(l+2-\mathrm{i} n, 2 l+3 ; z)= \\
& F(l+1-\mathrm{i} n, 2 l+1 ; z)-F(l+1-\mathrm{i} n, 2 l+2 ; z),
\end{aligned}
$$

Finally, the normalization factor for $E>0$ is found to be $A_{n 0}=\left[\frac{8 \pi / n}{1-e^{-2 \pi n}}\right]^{1 / 2}$, provided that the normalization $\int \psi_{n^{\prime} l m}^{*}(\rho) \psi_{n l m}(\rho) d^{3} r=2 \pi \delta\left(\frac{1}{n}-\frac{1}{n^{\prime}}\right)$ is used.

So many discussions on the analytical properties of the operator $\frac{1}{\hbar} A_{z}$. Let us turn our attention toward the other two, that is, $\frac{1}{\hbar} A_{ \pm}$, and ask whether we get any new properties out of the associated differential equations generated by them. To answer it, we take the differential operators $\frac{1}{\hbar} A_{ \pm}$given in eqn. (19) and act the both on the eigenfunctions $\psi_{n l m}(\rho)$. We arrive at

$$
\begin{aligned}
& \frac{1}{\hbar} A_{ \pm} \psi_{n l m}=2 A_{n l}\left\{\left[\frac{n}{2}-\frac{l(l+1)}{\rho}\right]\left(\mathrm{D}_{+}^{ \pm}(l, m) Y_{l+1}^{m+1}+\mathrm{D}_{-}^{ \pm}(l, m) Y_{l-1}^{m+1}\right)+(1 \pm m)\right. \\
& \left.\left(\mathrm{D}_{+}^{ \pm}(l, m) Y_{l+1}^{m+1}+\mathrm{D}_{-}^{ \pm}(l, m) Y_{l-1}^{m+1}\right) \partial_{\rho} \mp \mathrm{C}^{ \pm}(l, m)\left(\mathrm{D}_{+}^{0}(l, m \pm 1) Y_{l+1}^{m+1}+\mathrm{D}_{-}^{0}(l, m \pm 1) Y_{l-1}^{m+1}\right) \partial_{\rho}\right\} R_{n l} .
\end{aligned}
$$

Similar to the simplification procedure for $\frac{1}{\hbar} A_{z}$, both equations above can be done so. By combining the coefficients $\mathrm{D}_{ \pm}^{0}$ and $\mathrm{D}_{ \pm}^{0}$ into either $\mathrm{D}_{+}^{ \pm}(l, m)$ or $\mathrm{D}_{-}^{ \pm}(l, m)$ and eventually arranging all of them into either $\alpha_{ \pm}(l, m)$ or $\beta_{ \pm}(l, m)$, we at the end of the day come up with

$$
\frac{1}{\hbar} A_{ \pm} \psi_{n l m}=\alpha_{ \pm}(l, m) A_{n, l+1} R_{n, l+1} Y_{l+1}^{m \pm 1}+\beta_{ \pm}(l, m) A_{n, l-1} R_{n, l-1} Y_{l-1}^{m \pm 1} .
$$

See Appendix A for the combining details. Unfortunately, going from (38) to (39) reveals no new analytical features except for those ones that have been established previously for the operator $\frac{1}{\hbar} A_{z}$. In the other words, all three operators $\frac{1}{\hbar} A_{z}, \frac{1}{\hbar} A_{-}$and $\frac{1}{\hbar} A_{-}$are found to share the same amount of information as far as analytical perspective is concerned.

\section{Analytical Similarity in Two-Space}

The analytical structures presented in section 3 are not peculiar at all to the hydrogen-like atoms in three-dimensional space, we demonstrate in this section that some similarity also appear in the theory of two-dimensional hydrogen-like atoms.

In two-space, the Hamiltonian $H$ takes the exactly same form as its counterpart in three-space. The accidental symmetry in two-space is described by the z-component angular momentum $L_{z}$ and the twocomponent Runge-Lenz vector $\mathbf{R}=\left(R_{x}, R_{y}\right)$ defined exactly in eqn. (1). To simplify the presentations, we as usual rescale the vector $\mathbf{R}$ into $\mathbf{A}=\left(A_{x}, A_{y}\right)$ of the dimensions of $\hbar$ by the transformation (2) and then introduce the corresponding ladder-type operators: $A_{ \pm}=A_{x} \pm \mathrm{i} A_{y}$. With $A_{ \pm}$, the algebra of accidental symmetry becomes

$$
\left[L_{z}, A_{ \pm}\right]= \pm \hbar A_{ \pm}, \quad\left[A_{+}, A_{-}\right]=-( \pm) 2 \hbar L_{z},
$$

where the convention for the symbol $( \pm)$ is still applied. Apparently, the above commutation relations present an so $(2,1)$ symmetry for $\mathrm{E}>0$ and an $s o(3)$ symmetry for $\mathrm{E}<0$, precisely the symmetry of angular momentum in three-space.

The simultaneous eigenstates of mutually commuting operators $\left\{H, L_{z}, \mathbf{A}^{2}\right\}$ will be denoted by $\mid n, m>$, so that we have three eigenvalue equations: $H|n, m>=E| n, m>, L_{z}|n, m>=\hbar m| n, m>$, and

$$
\mathbf{A}^{2}\left|n, m>=\hbar^{2}\left[( \pm)\left(m^{2}+\frac{1}{4}\right)+n^{2}\right]\right| n, m>.
$$

Here, the energy eigenvalues $E$ is given in eqn. (6) with $n$ a real positive variable as usual for $\mathrm{E}>0$ but for $\mathrm{E}<0$ we have to choose $n=l+\frac{1}{2}$ for $l=0,1,2,3 \ldots$ It is worth mentioning that eqn. (41) comes from the operator identity: $\mathbf{A}^{2}=( \pm)\left(L_{z}^{2}+\frac{1}{4} \hbar^{2}\right)+\hbar^{2} n^{2}$ in two-space. Incorporating that identity with this one: $\mathbf{A}^{2}=A_{ \pm} A_{+} \pm( \pm) \hbar L_{z}$, we deduce algebraic equations for the ladder-type operators $A_{ \pm}$right away

$$
\frac{1}{\hbar} A_{ \pm}\left|n, m>=\left[n^{2}+( \pm)\left(m \pm \frac{1}{2}\right)^{2}\right]^{1 / 2}\right| n, m \pm 1>.
$$

In two-space, analytical properties of the eigenstates $\mid n, m>$ can be studied by projecting them into the plane-polar coordinates, $\mathbf{r}=(r, \varphi)$. To present the results, we likewise prefer to adopt the dimensionless position vector $\boldsymbol{\rho}=\tilde{\alpha} \mathbf{r}$, instead of $\mathbf{r}$. Consequently, the eigenfunctions $\psi_{n l m}(\rho)=<\boldsymbol{\rho} \mid n, m>$ admit the separation of variables

$$
\psi_{n m}(\rho)=\frac{1}{\sqrt{2 \pi}} A_{n m} R_{n m}(\rho) e^{\mathrm{i} m \phi},
$$

where $A_{n m}$ is the normalization factor that depends on both $n$ and $m$. forms

The ladder-type operators $A_{ \pm}$in the coordinates $\boldsymbol{\rho}=(\rho, \varphi)$ take the

$$
\frac{1}{\hbar} A_{ \pm}=-2 e^{ \pm i \phi}\left[\left(\partial_{\rho} \mp \frac{L_{z}}{\hbar}\right)\left(\frac{1}{2} \pm \frac{L_{z}}{\hbar}\right)+\frac{n}{2}\right] .
$$

now, acting the differential operators (44) on the eigenfunctions $\psi_{n m}(\boldsymbol{\rho})$ (43) and comparing the obtained result with the algebraic ones (42), we reach the following two valuable functional relations

$$
-2 A_{n m}\left[\left(\frac{1}{2} \pm m\right)\left(\partial_{\rho} \mp \frac{m}{\rho}\right)+\frac{n}{2}\right] R_{n m}=\left[n^{2}+( \pm)\left(m \pm \frac{1}{2}\right)^{2}\right]^{1 / 2} A_{n, m \pm 1} R_{n, m \pm 1} .
$$

Similar to that in three-space, there are two options for the choice 
of the factor $A_{n m}$. It again depends on whether $E<0$ or $E>0$.We shall examine the case of $E<0$ only, since the discussion for $E>0$ is closely analogous to that for $E<0$. The spectrum of negative energies is discrete and depends on the quantum number $n=l+\frac{1}{2}$. The degree of degeneracy of the $n$-th energy level is $2 l+1$ because $m$ can run from $-l$ to $+l$. The radial eigenfunctions for $\mathrm{E}<0$ are given by

$$
R_{n m}(\rho)=\rho^{|m|} e^{-\rho / 2} L_{n-|m|-1 / 2}^{2|m|}(\rho),
$$

where $L_{\alpha}^{\beta}(x)$ are the associated Laguerre polynomials. In the $E<0$ case, we set the normalization factor $A_{n m}$ to be

$$
A_{n m}=A_{n 0} \prod_{s=1}^{|m|}\left[n^{2}-\left(s-\frac{1}{2}\right)^{2}\right]^{-1 / 2}=\left[\frac{\left(n-|m|-\frac{1}{2}\right) !}{\left(n+|m|-\frac{1}{2}\right) !}\right]^{1 / 2},
$$

for both $m>0$ and $m<0$. Again, the product is replaced by unity for $m=0$. In addition, $A_{n 0}=\sqrt{\frac{\tilde{\alpha}^{2}}{2 n}}$, though it is not fixed by the algebraic method.

An important observation in eqns. (46) and (47) is that $R_{n m}$ and $A_{n m}$ both respect the reflection symmetry $m \rightarrow-m$. Interestingly, with the help of this symmetry, the upper equation in eqn. (45) gets transformed to the lower one. Therefore, one such equation is indeed enough. Let us take the upper equation and discuss the situations for $m>0$ and $m<0$ separately. We begin with the $m>0$ case.reena

1. In the case of $m>0$, we obtain the functional relation of $R_{n m}$, when plugging eqn. (47) into eqn. (45),

$$
\left[\left(\frac{1}{2}+m\right)\left(\partial_{\rho}-\frac{m}{\rho}\right)+\frac{n}{2}\right] R_{n m}=-\frac{1}{2} R_{n, m+1}
$$

now, the substitution of explicit form of the radial eigenfunctions $R_{n m}$ (46) into the above relation would certainly render another functional relation satisfied by the associated Laguerre polynomials $L_{n-m-\frac{1}{2}}^{2 m}(\rho)$. Surprisingly, the latter relation is exactly identical to that in eqn. (28), after the change of parameter $m \rightarrow l+\frac{1}{2}$ is made.

2. In the case of $m=-|m|<0$, we get after eqn. (47) is used

$$
\left[\left(\frac{1}{2}-|m|\right)\left(\partial_{\rho}+\frac{|m|}{\rho}\right)+\frac{n}{2}\right] R_{n m}=-\frac{1}{2}\left[n^{2}-\left(|m|-\frac{1}{2}\right)^{2}\right] R_{n, m+1} .
$$

then, the substitution of $R_{n m}$ (46) into this relation results in another functional relation satisfied by $L_{n-|m|-\frac{1}{2}}^{2|m|}(\rho)$, from which eqn. (29) can be directly recovered by the substitution $|m| \rightarrow l+\frac{1}{2}$.

Both notable functional relations (28) and (29) established from accidental symmetry also appear in the hydrogen-like atoms in twodimensional space.

\section{Conclusion}

In this paper, we study accidental symmetry of the hydrogenlike atoms, based on the time-independent Schrödinger equation. The rescaled Runge-Lenz vectorial operator is adopted to reduce cumbersome notations. The algebra described by the angular momentum $\mathbf{L}$ and Runge-Lenz operator $\mathbf{A}$ is briefly reviewed.
The representation of eigenstates for the commuting operators $\left\{H, \mathbf{L}^{2}, L_{z}, \mathbf{A}^{2}\right\}$ are inspected, as well. Acted on by the ladder-type operators such as $A_{ \pm}$and $A_{z}$ analytical properties of the eigenfunctions $\psi_{n l m}(\rho)$ constructed in the dimensionless spherical coordinates are accordingly deduced, especially for both bound states and continuous states. Some unfamiliar functional relations of the radial eigenfunctions of the Hamiltonian are established. Similar analytical properties shared by both two-dimensional and three-dimensional hydrogen atoms are illustrated and analyzed. To conclude, it seems that functional relations in both equs. (28) and (29) for the radial eigenfunctions are quite general in the theories of hydrogen-like atoms. Though it is not shown here, same conclusion will be reached for eqns. (35) and (36), too.

\section{References}

1. Pauli W (1926) About the hydrogen spectrum from the standpoint of the new quantum mechanics. Z Phys 36: 336-363.

2. Fock VA (1935) Hydrogen atom and non-Euclidean geometry. Z Phys 98: 169188.

3. Bargmann V (1936) The theory of the hydrogen atom. Z Phys 99: 576-582.

4. Barut AO (1964) Electrodynamics and classical theory of fields \& particle. Phys Rev 135: B839.

5. Malkin IA, Man'ko VI (1965) Symmetry of the hydrogen atom. JEPT Lett 2 146-50.

6. Bander M, Itzykson C (1966) Group Theory and the Hydrogen Atom (I). Rev Mod Phys 38: 330

7. Nambu Y, Hara Y (1966) Nonleptonic Decays of K Mesons. Physical Review Letters 16: 875.

8. Barut AO, Kleinert $\mathrm{H}$ (1967) Transition probabilities of the hydrogen atom from noncompact dynamical Groups. Phys Rev 156: 1541.

9. Fronsdal C (1967) Infinite Multiplets and the Hydrogen Atom. Phys Rev 156: 1665.

10. Nambu Y (1967) Infinite-Component Wave Equations with Hydrogenlike Mass Spectra. Phys Rev 160: 1171.

11. Kleinert HM (1968) A model for deep inelastic structure functions. Nuclear Functions B 43: 301-330.

12. Infeld L, Hull TE (1951) The Factorization Method. Rev Mod Phys 23: 21.

13. Lanik J (1967) Dynamical algebra and potentials. Nuc Phys B2: 263-266.

14. Ferreira PL (1969) On the Ladder Representations of the $0(4,2)$ Group. Rev Mex Fis 18: 233.

15. Englefield MJ (1972) Group Theory and the Coulomb Problem. Wiley New York.

16. Runge C (1919) Studies in Mathematical Physics: Essays in Honor of Valentine Bargmann. Princeton University Press.

17. Lenz W (2005) Relativistic trajectories and the Runge-Lenz vector. American Journal of Physics 73: 581.

18. Wulfman CE (1971) Dynamical Groups in Atomic and Molecular Physics. Recent Advances in Group Theory and Their Application to Spectroscopy, pp 329-403.

19. Pauli W (1965) Nuclear Physics. Results of the Exact Natural Sciences 37: 85.

20. Schwinger J (1965) On Angular Momentum.Quantum Thoery of Angular Momentum. World Scientific, USA.

21. Arfken GB, Weber HJ (2000) Mathematical Methods for Physicists.Academic Press. 